Supplement of Hydrol. Earth Syst. Sci., 22, 1735-1748, 2018

https://doi.org/10.5194/hess-22-1735-2018-supplement

(C) Author(s) 2018. This work is distributed under

the Creative Commons Attribution 3.0 License.

(c) (1)

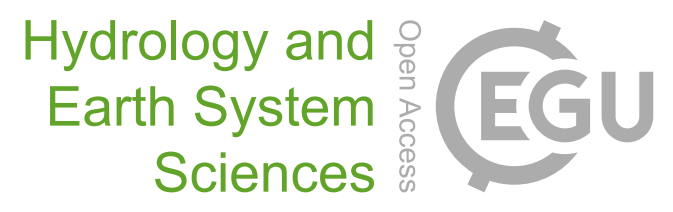

Supplement of

\title{
Scaling properties reveal regulation of river flows in the Amazon through a "forest reservoir"
}

\section{Juan Fernando Salazar et al.}

Correspondence to: Juan Fernando Salazar (juan.salazar@udea.edu.co)

The copyright of individual parts of the supplement might differ from the CC BY 3.0 License. 


\section{Contents}

1. River flow data: Table $\mathrm{S} 1$

2. Estimation of power laws: Figures S1 to S6, and Table S2.

3. Tests for the difference between the values of the scaling exponents and the critical value 1: Tables S3 to S8.

$5 \quad 4$. Scaling results using $A$ as the scale parameter: Figures S7-S9, Tables S9-S15.

\section{River flow data}

Details about the river flow records and gauges used in this study are given in Supplementary Table S1. The locations of the 85 gauges is indicated in Figure 1. Mean and extreme river flows were computed from daily records. Any year with less than 320 daily records was discarded. The lengths of the records that were used in the calculations vary between 26 and 47 years, with 10 an average of 38 years. Drainage areas vary between $1,617 \mathrm{~km}^{2}$ at Pari Cachoeira and 4,680,000 $\mathrm{km}^{2}$ at Obidos. 
Table S1: River flow gauges provided by the SO-HYBAM project (formerly ORE-HYBAM project, www.ore-hybam.org).

\begin{tabular}{|c|c|c|c|c|c|c|c|c|c|}
\hline ID & Code & Name & River & Initial date & Final date & $\begin{array}{l}\text { Record } \\
\text { length } \\
(\mathrm{yr})\end{array}$ & $\begin{array}{l}\text { Lat. } \\
\left({ }^{\circ}\right)\end{array}$ & $\begin{array}{l}\text { Lon. } \\
\left({ }^{\circ}\right)\end{array}$ & $\begin{array}{l}\text { Drainage } \\
\text { area } \\
\left(\mathrm{km}^{2}\right)\end{array}$ \\
\hline 1 & 14300000 & $\begin{array}{l}\text { Pari } \quad \text { Ca- } \\
\text { choeira }\end{array}$ & Tiquie & $7 / 7 / 1980$ & $4 / 29 / 2014$ & 34 & 0.25 & -69.78 & 1,617 \\
\hline 2 & 18250000 & Uruara & $\begin{array}{l}\text { Para do } \\
\text { Uruara }\end{array}$ & $12 / 14 / 1978$ & $4 / 29 / 2014$ & 36 & -3.68 & -53.55 & 2,628 \\
\hline 3 & 14540000 & $\begin{array}{l}\text { Fazenda Ban- } \\
\text { deira Branca }\end{array}$ & Cotingo & $10 / 2 / 1970$ & $4 / 29 / 2014$ & 44 & 4.63 & -60.47 & 3,075 \\
\hline 4 & 15050000 & $\begin{array}{l}\text { Pontes e Lac- } \\
\text { erda }\end{array}$ & Guapore & $8 / 21 / 1971$ & $7 / 30 / 2014$ & 43 & -15.22 & -59.35 & 3,140 \\
\hline 5 & 15565000 & Jaru & Jaru & $6 / 22 / 1981$ & $3 / 30 / 2012$ & 31 & -10.45 & -62.47 & 3,965 \\
\hline 6 & 14310000 & Cunuri & Tiquie & $8 / 15 / 1982$ & $4 / 29 / 2014$ & 32 & 0.21 & -69.38 & 4,198 \\
\hline 7 & 14220000 & Louro Poco & Aiari & $8 / 2 / 1982$ & $10 / 30 / 2008$ & 26 & 1.34 & -68.69 & 4,708 \\
\hline 8 & 17230000 & $\begin{array}{l}\text { Lucas do Rio } \\
\text { Verde }\end{array}$ & Verde & $8 / 17 / 1973$ & $4 / 16 / 2007$ & 34 & -13.05 & -55.90 & 5,327 \\
\hline 9 & 14550000 & $\begin{array}{l}\text { Maloca do } \\
\text { Contao }\end{array}$ & Cotingo & $9 / 5 / 1975$ & $4 / 29 / 2014$ & 39 & 4.17 & -60.53 & 5,815 \\
\hline 10 & 18280000 & Apalai & $\begin{array}{l}\text { Paru de } \\
\text { Este }\end{array}$ & $7 / 25 / 1980$ & $9 / 29 / 2012$ & 32 & 1.22 & -54.66 & 5,902 \\
\hline 11 & 15750000 & Humboldt & Aripuana & $5 / 25 / 1979$ & $6 / 29 / 2014$ & 35 & -10.17 & -59.46 & 6,653 \\
\hline 12 & 15430000 & Ariquemes & Jamari & $7 / 16 / 1970$ & $5 / 30 / 2014$ & 44 & -9.93 & -63.06 & 7,295 \\
\hline 13 & 14526000 & Bom Fim & Tacutu & 7/7/1984 & $4 / 29 / 2014$ & 30 & 3.38 & -59.82 & 9,744 \\
\hline 14 & 12360000 & Foz do Breu & Jurua & $6 / 21 / 1982$ & $6 / 1 / 2012$ & 30 & -9.40 & -72.70 & 10,141 \\
\hline 15 & 14350000 & $\begin{array}{l}\text { Jusante da } \\
\text { Cachoeira do } \\
\text { Caju }\end{array}$ & Curicuriari & $6 / 2 / 1982$ & $3 / 30 / 2014$ & 32 & -0.25 & -67.01 & 10,228 \\
\hline 16 & 13550000 & Xapuri & Acre & 2/17/1968 & $1 / 30 / 2014$ & 46 & -10.65 & -68.51 & 11,765 \\
\hline 17 & 15558000 & $\begin{array}{l}\text { Pimenta } \\
\text { Bueno }\end{array}$ & Apedia & $5 / 30 / 1980$ & $7 / 30 / 2014$ & 34 & -11.68 & -61.19 & 12,346 \\
\hline 18 & 15550000 & Santa Isabel & Candeias & $3 / 10 / 1976$ & $6 / 29 / 2014$ & 38 & -8.80 & -63.71 & 12,640 \\
\hline
\end{tabular}

- Continues in the next page - 
- Come from previous page -

\begin{tabular}{|c|c|c|c|c|c|c|c|c|c|}
\hline 19 & 14680001 & $\begin{array}{l}\text { Fe d Esper- } \\
\text { anca }\end{array}$ & Mucajai & $12 / 8 / 1973$ & $4 / 29 / 2014$ & 41 & 2.87 & -61.44 & 13,658 \\
\hline 20 & 12880000 & $\begin{array}{l}\text { Estirao da } \\
\text { Santa Cruz }\end{array}$ & Tefe & 2/10/1981 & $3 / 30 / 2014$ & 33 & -4.29 & -65.20 & 13,708 \\
\hline 21 & 15910000 & Sucunduri & Sucunduri & $10 / 24 / 1973$ & $9 / 29 / 2013$ & 40 & -6.80 & -59.04 & 13,938 \\
\hline 22 & 14440000 & $\begin{array}{l}\text { Posto Ajuri- } \\
\text { caba }\end{array}$ & Demeni & $5 / 29 / 1982$ & $4 / 29 / 2014$ & 32 & 0.88 & -62.62 & 14,756 \\
\hline 23 & 12650000 & Feijo & Envira & $9 / 26 / 1980$ & $5 / 19 / 2012$ & 32 & -8.15 & -70.37 & 15,329 \\
\hline 24 & 12370000 & Taumaturgo & Jurua & 4/14/1981 & $5 / 31 / 2012$ & 31 & -8.93 & -72.78 & 16,581 \\
\hline 25 & 18200000 & Arapari & Maicuru & 7/1/1972 & $2 / 27 / 2014$ & 42 & -1.78 & -54.40 & 17,072 \\
\hline 26 & 15120001 & $\begin{array}{l}\text { Vila Bela } \\
\text { da Santis } \\
\text { Trindade }\end{array}$ & Guapore & 7/1/1976 & $7 / 30 / 2014$ & 38 & -15.01 & -59.95 & 18,412 \\
\hline 27 & 14230000 & Missao Icana & Icana & 7/17/1980 & $3 / 30 / 2014$ & 34 & 1.07 & -67.59 & 22,282 \\
\hline 28 & 17090000 & $\begin{array}{l}\text { Boca do In- } \\
\text { ferno }\end{array}$ & Curua & $3 / 29 / 1973$ & $9 / 29 / 2013$ & 40 & -1.50 & -54.87 & 22,500 \\
\hline 29 & 13600002 & Rio Branco & Acre & $8 / 9 / 1967$ & $5 / 30 / 2014$ & 47 & -9.98 & -67.80 & 22,670 \\
\hline 30 & 16500000 & $\begin{array}{l}\text { Estirao da } \\
\text { Angelica }\end{array}$ & Mapuera & $11 / 22 / 1970$ & $4 / 29 / 2014$ & 44 & -1.10 & -57.06 & 26,040 \\
\hline 31 & 15560000 & Ji-Parana & Jiparana & $12 / 16 / 1977$ & $7 / 30 / 2014$ & 37 & -10.87 & -61.94 & 33,012 \\
\hline 32 & 13650000 & $\begin{array}{l}\text { Floriano } \\
\text { Peixoto }\end{array}$ & Acre & $7 / 26 / 1967$ & $4 / 29 / 2014$ & 47 & -9.05 & -67.37 & 33,469 \\
\hline 33 & 18650000 & Cajueiro & Curua & $11 / 20 / 1975$ & $11 / 29 / 2011$ & 36 & -5.65 & -54.52 & 34,693 \\
\hline 34 & 17120000 & $\begin{array}{l}\text { Porto dos } \\
\text { Gauchos }\end{array}$ & Arinos & $9 / 15 / 1973$ & $5 / 30 / 2014$ & 41 & -11.54 & -57.42 & 36,913 \\
\hline 35 & 14495000 & $\begin{array}{l}\text { Fazenda } \\
\text { Cajupiranga }\end{array}$ & Uraricoera & $8 / 10 / 1979$ & $3 / 30 / 2014$ & 35 & 3.44 & -61.04 & 37,430 \\
\hline 36 & 13886000 & Bacaba & Cuniua & $12 / 15 / 1979$ & $3 / 20 / 2014$ & 35 & -6.32 & -64.88 & 38,270 \\
\hline 37 & 12500000 & $\begin{array}{l}\text { Cruzeiro do } \\
\text { Sul }\end{array}$ & Jurua & $8 / 24 / 1967$ & $6 / 4 / 2012$ & 45 & -7.61 & -72.68 & 38,537 \\
\hline 38 & 17280000 & Cachoeirao & $\begin{array}{l}\text { Teles } \\
\text { Pires }\end{array}$ & $11 / 18 / 1975$ & $7 / 30 / 2014$ & 39 & -11.65 & -55.70 & 39,000 \\
\hline 39 & 14260000 & Uaracu & Uaupes & $12 / 3 / 1977$ & $11 / 29 / 2011$ & 34 & 0.48 & -69.13 & 40,506 \\
\hline
\end{tabular}

- Continues in the next page - 
- Come from previous page -

\begin{tabular}{|c|c|c|c|c|c|c|c|c|c|}
\hline 40 & 18500000 & $\begin{array}{l}\text { Boa Esper- } \\
\text { anca }\end{array}$ & Fresco & $1 / 23 / 1976$ & $11 / 29 / 2013$ & 37 & -6.72 & -51.78 & 43,030 \\
\hline 41 & 14280001 & Taraqua & Uaupes & $5 / 23 / 1977$ & $4 / 29 / 2014$ & 37 & 0.13 & -68.54 & 44,732 \\
\hline 42 & 15800000 & $\begin{array}{l}\text { Boca do } \\
\text { Guariba }\end{array}$ & Aripuana & $12 / 12 / 1977$ & $12 / 30 / 2011$ & 34 & -7.68 & -60.30 & 47,773 \\
\hline 43 & 15130000 & Pimenteiras & Guapore & $6 / 26 / 1983$ & $7 / 30 / 2014$ & 31 & -13.48 & -61.05 & 50,055 \\
\hline 44 & 14515000 & $\begin{array}{l}\text { Fazenda Pas- } \\
\text { sarao }\end{array}$ & Uraricoera & $5 / 2 / 1977$ & $2 / 27 / 2014$ & 37 & 3.21 & -60.57 & 50,985 \\
\hline 45 & 19150000 & Sao Francisco & Jari & $7 / 2 / 1972$ & $3 / 21 / 2014$ & 42 & -0.57 & -52.58 & 51,340 \\
\hline 46 & 17093000 & Fontanilhas & Juruena & $1 / 27 / 1978$ & $6 / 29 / 2014$ & 36 & -11.36 & -58.34 & 52,200 \\
\hline 47 & 12520000 & Ipixuna & Jurua & 10/10/1981 & $6 / 14 / 2012$ & 31 & -7.05 & -71.68 & 55,806 \\
\hline 48 & 15580000 & Tabajara & Jiparana & $12 / 13 / 1977$ & $5 / 30 / 2014$ & 37 & -8.93 & -62.05 & 60,212 \\
\hline 49 & 14110000 & Cucui & Negro & $7 / 23 / 1980$ & $4 / 29 / 2014$ & 34 & 1.22 & -66.85 & 61,781 \\
\hline 50 & 13410000 & $\begin{array}{l}\text { Seringal da } \\
\text { Caridade }\end{array}$ & Purus & $8 / 2 / 1967$ & $4 / 29 / 2014$ & 47 & -9.04 & -68.57 & 63,166 \\
\hline 51 & 17340000 & Indeco & $\begin{array}{l}\text { Teles } \\
\text { Pires }\end{array}$ & $11 / 2 / 1975$ & $8 / 30 / 2014$ & 39 & -10.11 & -55.57 & 64,650 \\
\hline 52 & 12550000 & $\begin{array}{l}\text { Eirunepe- } \\
\text { Montante }\end{array}$ & Jurua & 2/23/1979 & $6 / 20 / 2012$ & 33 & -6.68 & -69.90 & 77,136 \\
\hline 53 & 13710001 & Valparaiso & Purus & $2 / 27 / 1975$ & $4 / 29 / 2014$ & 39 & -8.68 & -67.40 & 103,285 \\
\hline 54 & 15830000 & Prainha Velha & Aripuana & $6 / 3 / 1974$ & $9 / 29 / 2013$ & 39 & -7.25 & -60.40 & 108,578 \\
\hline 55 & 15150000 & Pedras Negras & Guapore & $12 / 18 / 1980$ & $7 / 30 / 2014$ & 34 & -12.85 & -62.90 & 109,788 \\
\hline 56 & 14250000 & Sao Felipe & Negro & $12 / 9 / 1977$ & $4 / 29 / 2014$ & 37 & 0.37 & -67.31 & 110,862 \\
\hline 57 & 14710000 & Caracarai & Branco & $1 / 2 / 1967$ & $5 / 30 / 2014$ & 47 & 1.82 & -61.12 & 124,980 \\
\hline 58 & 12700000 & $\begin{array}{l}\text { Santos Du- } \\
\text { mont }\end{array}$ & Jurua & 4/17/1981 & $5 / 30 / 2014$ & 33 & -6.44 & -68.24 & 142,234 \\
\hline 59 & 17420000 & Tres Marias & $\begin{array}{l}\text { Teles } \\
\text { Pires }\end{array}$ & $12 / 2 / 1975$ & $3 / 30 / 2014$ & 39 & -7.61 & -57.95 & 144,400 \\
\hline 60 & 12840000 & Gaviao & Jurua & $6 / 23 / 1972$ & $1 / 30 / 2014$ & 42 & -4.84 & -66.85 & 162,000 \\
\hline 61 & 14330000 & Curicuriari & Negro & $11 / 28 / 1977$ & $3 / 30 / 2014$ & 37 & -0.20 & -66.80 & 194,462 \\
\hline 62 & 12845000 & $\begin{array}{l}\text { Vila Bitten- } \\
\text { court }\end{array}$ & Japura & $1 / 27 / 1980$ & $2 / 27 / 2014$ & 34 & -1.40 & -69.42 & 197,136 \\
\hline 63 & 18460000 & Boa Sorte & Xingu & $1 / 24 / 1977$ & $11 / 29 / 2013$ & 36 & -6.75 & -51.98 & 206,863 \\
\hline
\end{tabular}

- Continues in the next page - 
- Come from previous page -

\begin{tabular}{|c|c|c|c|c|c|c|c|c|c|}
\hline 64 & 13870000 & Labrea & Purus & $7 / 5 / 1967$ & $5 / 30 / 2014$ & 47 & -7.25 & -64.80 & 220,351 \\
\hline 65 & 13880000 & Canutama & Purus & $1 / 2 / 1973$ & $3 / 30 / 2014$ & 41 & -6.53 & -64.38 & 230,012 \\
\hline 66 & 12850000 & Acanaui & Japura & 7/9/1973 & $2 / 27 / 2014$ & 41 & -1.82 & -66.60 & 242,259 \\
\hline 67 & 14420000 & Serrinha & Negro & $11 / 30 / 1967$ & $3 / 30 / 2014$ & 47 & -0.48 & -64.83 & 279,945 \\
\hline 68 & 17430000 & $\begin{array}{l}\text { Barra Do } \\
\text { Sao Manuel } \\
\text { Jusante }\end{array}$ & Tapajos & $11 / 2 / 1975$ & $3 / 30 / 2014$ & 39 & -7.34 & -58.16 & 330,900 \\
\hline 69 & 15200000 & $\begin{array}{l}\text { Principe } \mathrm{Da} \\
\text { Beira }\end{array}$ & Guapore & $4 / 22 / 1983$ & $7 / 30 / 2014$ & 31 & -12.43 & -64.43 & 342,833 \\
\hline 70 & 17650000 & Jatoba & Tapajos & $12 / 21 / 1972$ & $4 / 14 / 2012$ & 40 & -5.15 & -56.85 & 389,300 \\
\hline 71 & 18850000 & Altamira & Xingu & 6/6/1968 & $4 / 29 / 2014$ & 46 & -3.21 & -52.21 & 446,203 \\
\hline 72 & 17730000 & Itaituba & Tapajos & 2/13/1968 & $3 / 30 / 2014$ & 46 & -4.28 & -55.98 & 451,600 \\
\hline 73 & 15250000 & $\begin{array}{l}\text { Guajara- } \\
\text { Mirim }\end{array}$ & Mamore & 8/9/1970 & $6 / 29 / 2014$ & 44 & -10.79 & -65.35 & 589,497 \\
\hline 74 & 15320002 & Abuna & Madeira & $6 / 28 / 1976$ & $6 / 29 / 2014$ & 38 & -9.70 & -65.36 & 899,761 \\
\hline 75 & 15400000 & Porto Velho & Madeira & $4 / 11 / 1967$ & $1 / 30 / 2014$ & 47 & -8.74 & -63.92 & 954,285 \\
\hline 76 & 11400000 & $\begin{array}{l}\text { Sao Paulo de } \\
\text { Olivenca }\end{array}$ & Solimoes & $7 / 19 / 1973$ & $12 / 30 / 2011$ & 38 & -3.45 & -68.75 & 990,781 \\
\hline 77 & 15630000 & Humaita & Madeira & 4/19/1967 & $4 / 13 / 2014$ & 47 & -7.51 & -63.02 & $1,066,240$ \\
\hline 78 & 15700000 & Manicore & Madeira & $4 / 25 / 1967$ & $2 / 27 / 2014$ & 47 & -5.82 & -61.30 & $1,123,670$ \\
\hline 79 & 11500000 & $\begin{array}{l}\text { Santo An- } \\
\text { tonio } \\
\text { Ica }\end{array}$ & Solimoes & $7 / 15 / 1973$ & $2 / 27 / 2014$ & 41 & -3.08 & -67.93 & $1,134,540$ \\
\hline 80 & 15860000 & $\begin{array}{l}\text { Fazenda Vista } \\
\text { Alegre }\end{array}$ & Madeira & $5 / 3 / 1967$ & $2 / 27 / 2014$ & 47 & -4.90 & -60.03 & $1,324,730$ \\
\hline 81 & 13150000 & Itapeua & Solimoes & $4 / 5 / 1971$ & $1 / 30 / 2014$ & 43 & -4.06 & -63.03 & $1,769,000$ \\
\hline 82 & 14100000 & Manacapuru & Solimoes & $6 / 29 / 1972$ & $1 / 30 / 2014$ & 42 & -3.31 & -60.61 & $2,147,740$ \\
\hline 83 & 15040000 & Careiro & $\begin{array}{l}\text { Parana do } \\
\text { Careiro }\end{array}$ & 8/30/1977 & $2 / 27 / 2014$ & 37 & -3.20 & -59.82 & $2,853,080$ \\
\hline 84 & 15030000 & Jatuarana & Amazonas & 8/27/1977 & $2 / 27 / 2014$ & 37 & -3.05 & -59.68 & $2,854,290$ \\
\hline 85 & 17050000 & Obidos & Amazonas & 2/23/1968 & $1 / 30 / 2015$ & 47 & -1.95 & -55.51 & $4,680,000$ \\
\hline
\end{tabular}




\section{Estimation of power laws}

The scaling relations given by power laws $E\left[Q^{k}\right]=\gamma A^{\delta}$ (equivalent to Equation (1) with $S=A$ ) and $E\left[Q^{k}\right]=\alpha L A^{\beta}$ (equivalent to Equation (7)), respectively, are equivalent to the linear functions

$\ln \left(E\left[Q^{k}\right]\right)=\ln (\gamma)+\delta \ln (A)$

5

and

$\ln \left(E\left[Q^{k}\right]\right)=\ln (\alpha)+\beta \ln (L A)$,

where the scaling exponent ( $\delta$ or $\beta$ ) and coefficient ( $\gamma$ or $\alpha$ ) can be estimated from linear regressions between $\ln \left(E\left[Q^{k}\right]\right)$ and either $\ln (A)$ or $\ln (L A) . E\left[Q^{k}\right]$ is the $k$ th order statistical moment of the probability distribution function of mean or extreme river flows, $A$ is the drainage area, and $L A$ is the cumulative leaf area of the river basin. These linear regressions were computed through standard least-squares methods. The statistical moments $E\left[Q^{k}\right]$ were estimated from time series of annual mean and extreme (floods and low flows) river flows obtained from the daily records described in Table S1. The cumulative leaf area associated with each gauge was estimated as $L A=A \times \overline{L A I}$ where $\overline{L A I}$ is the long-term (1981-2012) average leaf area index spatially averaged over $A$, using published data of annual $L A I$. Figure 1 shows the resulting $\overline{L A I}$ map.

Figures S1 to S6 show that mean flows, low flows and floods, scale with drainage area (we will refer to this as hydrological scaling) as well as with leaf area (ecohydrological scaling), in the whole Amazon river basin (Fig. S1) and in its five major sub-basins treated as independent systems: the Negro, Solimoes, Madeira, Tapajos and Xingu (Figs. S2-S6). 

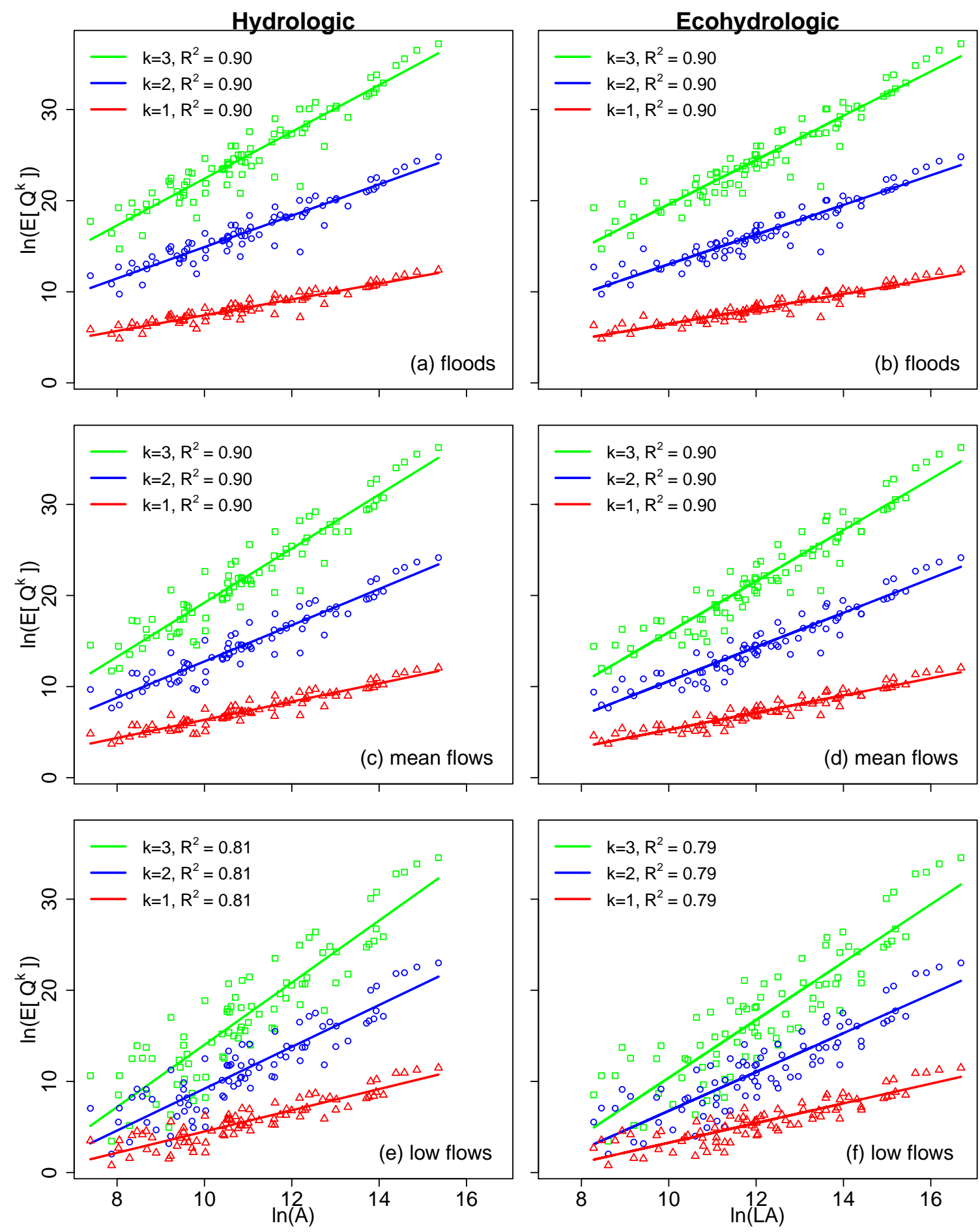

Figure S1. Hydrologic (left) and ecohydrologic (right) scaling power-law relations for floods (top), mean flows (middle) and low flows (bottom), in the whole Amazon basin, for different orders $(k)$ of the statistical moments. 

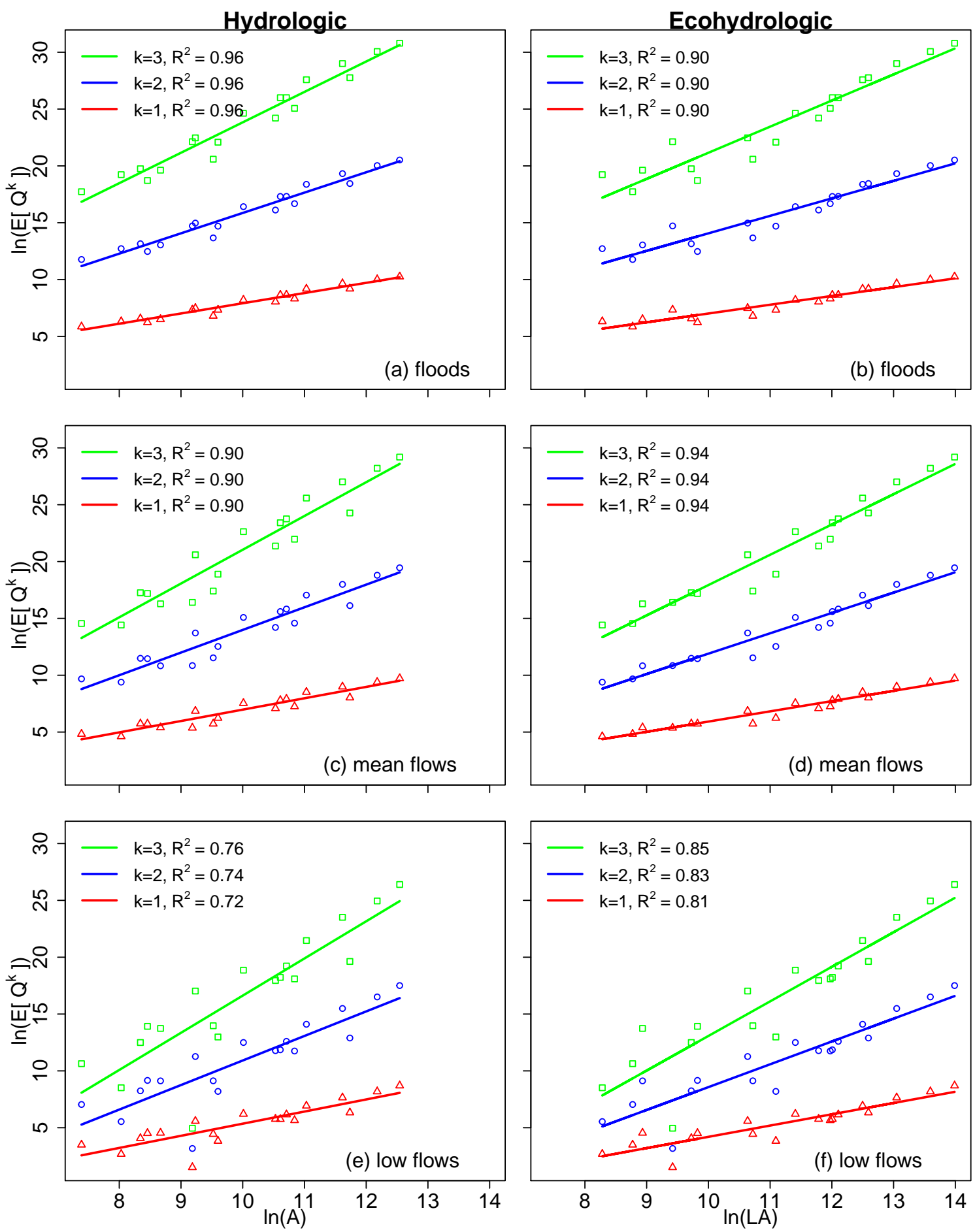

Figure S2. Same as Fig. S1 but for the Negro basin. 

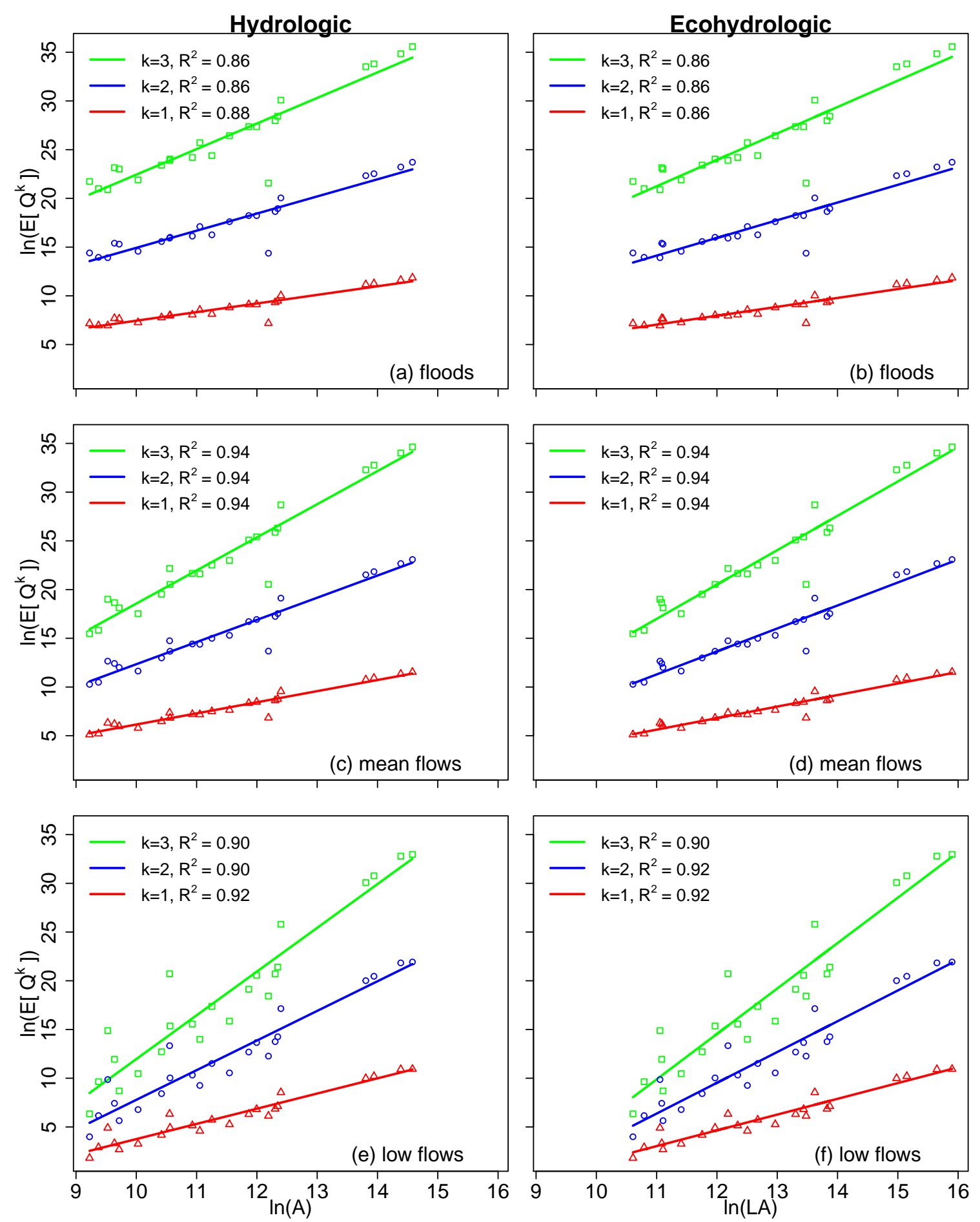

Figure S3. Same as Fig. S1 but for the Solimoes basin. 

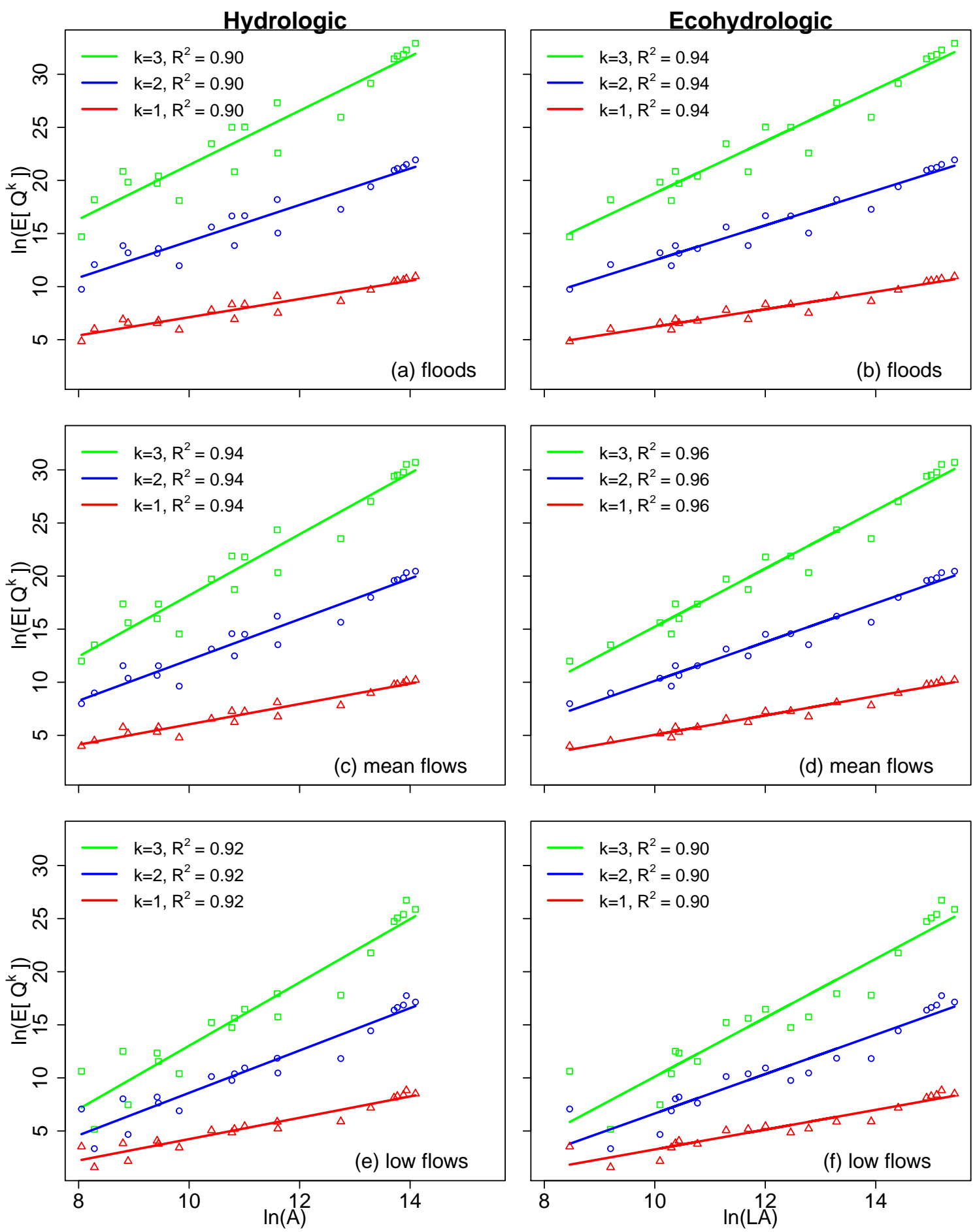

Figure S4. Same as Fig. S1 but for the Madeira basin. 

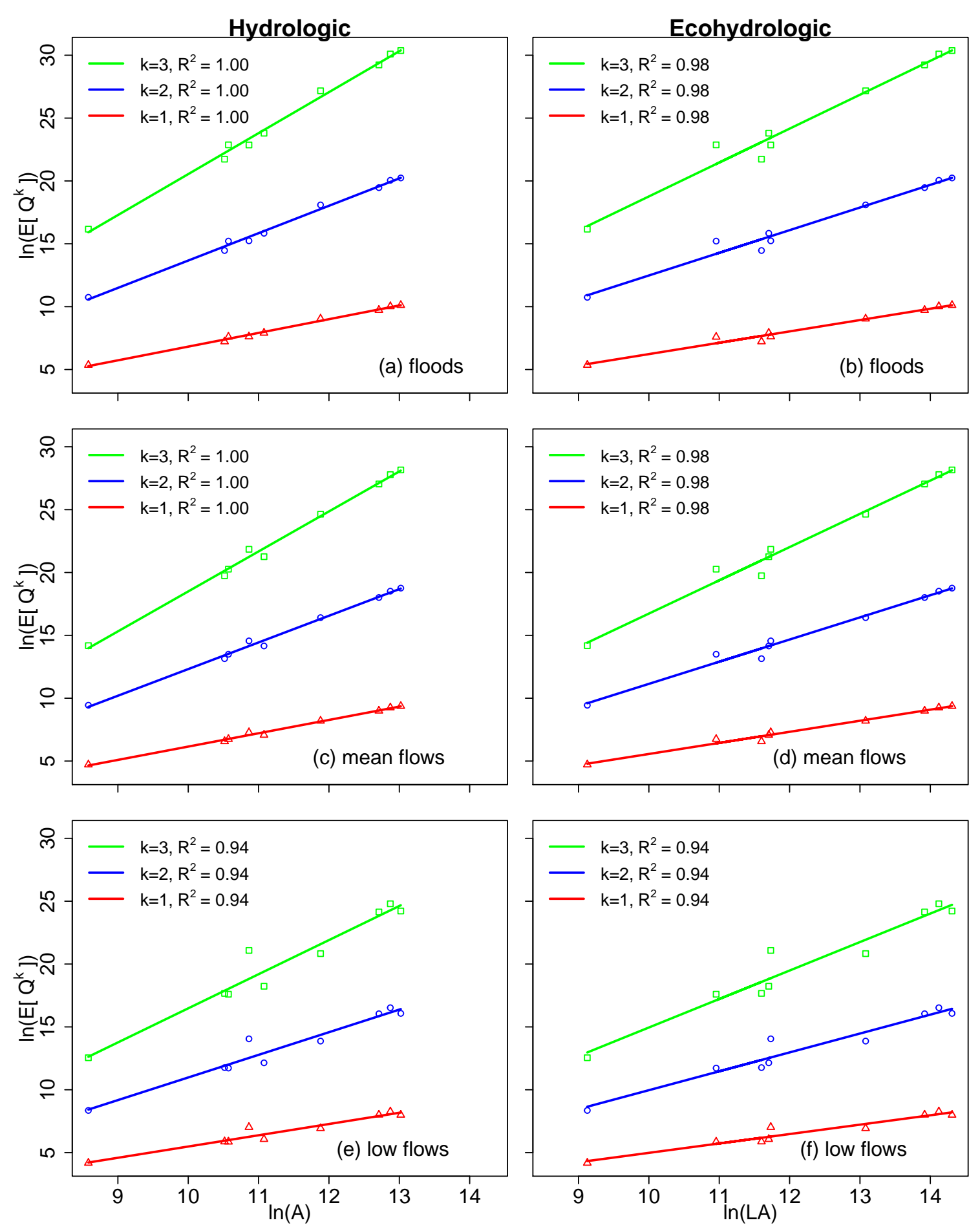

Figure S5. Same as Fig. S1 but for the Tapajos basin. 

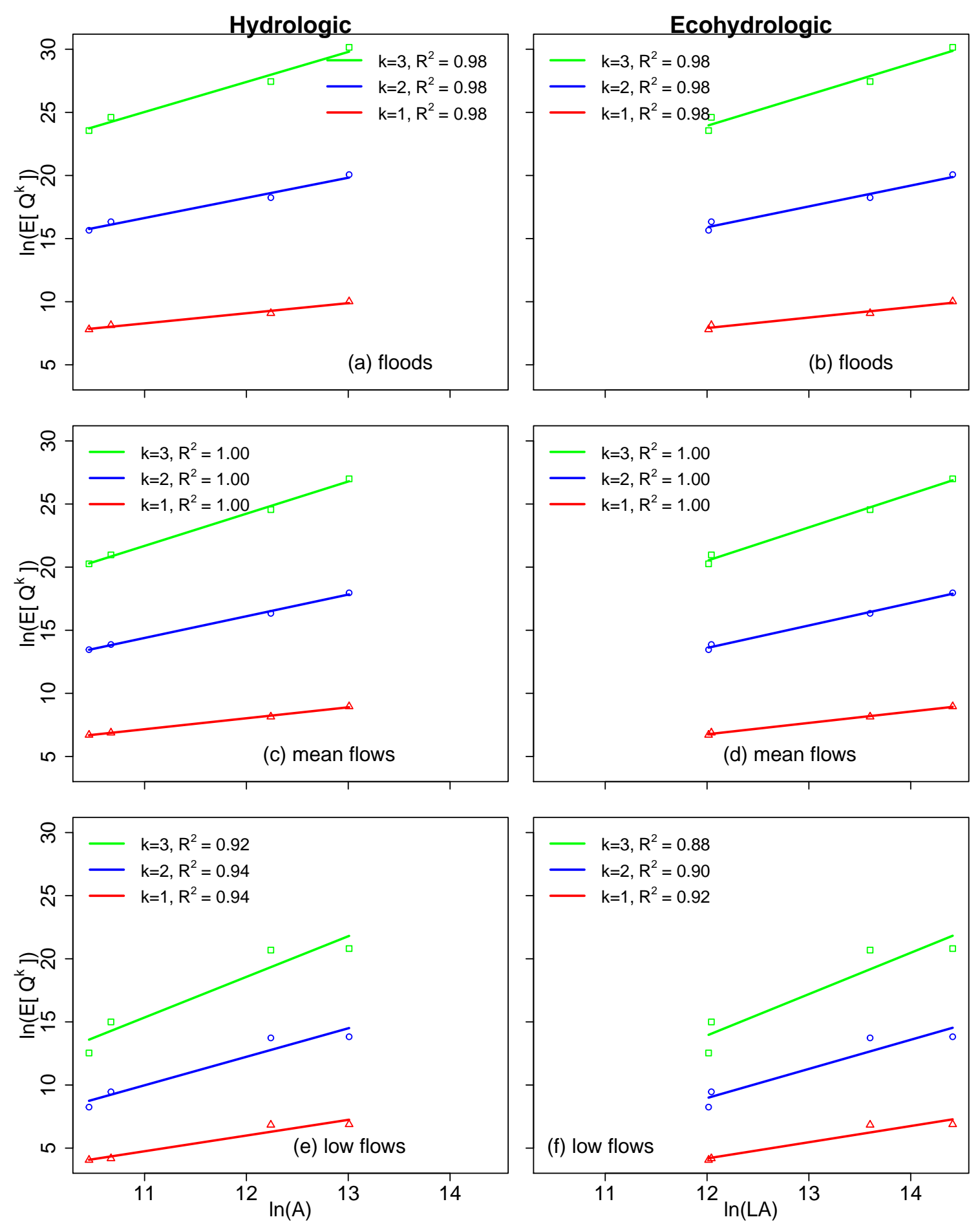

Figure S6. Same as Fig. S1 but for the Xingu basin. 
The statistical significance of the ecohydrological scaling (Equation (7)) was evaluated through the determination and correlation coefficients ( $R^{2}$ and $r$, Fig. 1 and Figs. S1-S6), as well as by testing the hypothesis that $\ln \left(E\left[Q^{k}\right]\right)$ and $\ln (L A)$ are not linearly related, i.e. the null hypothesis $H_{0}: \beta=0$, through standard $t$-tests. All the $t$-tests show that $\ln \left(E\left[Q^{k}\right]\right)$ and $\ln (L A)$ have a statistically significant $(p<0.05)$ linear relation (Table $\mathbf{S} 2)$.

Table S2. $t$-tests for the significance of the regressions in the ecohydrological scaling for the first statistical moment of river flows. In all cases the null hypothesis, $H_{0}$ is rejected, so the alternative hypothesis, $H_{a}$, is accepted.

\begin{tabular}{llccccc}
\hline Basin & Q type & $H_{0}$ & $H_{a}$ & $p$-value & Test result & Conclusion \\
\hline \multirow{4}{*}{ Amazon } & Flood & $\beta_{F}=0$ & $\beta_{F} \neq 0$ & $1.60 \mathrm{E}-43$ & reject $H_{0}$ & $\beta_{F} \neq 0$ \\
& Mean flow & $\beta_{M}=0$ & $\beta_{M} \neq 0$ & $4.30 \mathrm{E}-44$ & reject $H_{0}$ & $\beta_{M} \neq 0$ \\
& Low flow & $\beta_{L}=0$ & $\beta_{L} \neq 0$ & $4.14 \mathrm{E}-29$ & reject $H_{0}$ & $\beta_{L} \neq 0$ \\
\hline \multirow{4}{*}{ Negro } & Flood & $\beta_{F}=0$ & $\beta_{F} \neq 0$ & $2.12 \mathrm{E}-10$ & reject $H_{0}$ & $\beta_{F} \neq 0$ \\
& Mean flow & $\beta_{M}=0$ & $\beta_{M} \neq 0$ & $5.05 \mathrm{E}-12$ & reject $H_{0}$ & $\beta_{M} \neq 0$ \\
& Low flow & $\beta_{L}=0$ & $\beta_{L} \neq 0$ & $1.60 \mathrm{E}-07$ & reject $H_{0}$ & $\beta_{L} \neq 0$ \\
\hline \multirow{4}{*}{ Solimoes } & Flood & $\beta_{F}=0$ & $\beta_{F} \neq 0$ & $9.54 \mathrm{E}-11$ & reject $H_{0}$ & $\beta_{F} \neq 0$ \\
& Mean flow & $\beta_{M}=0$ & $\beta_{M} \neq 0$ & $6.17 \mathrm{E}-14$ & reject $H_{0}$ & $\beta_{M} \neq 0$ \\
& Low flow & $\beta_{L}=0$ & $\beta_{L} \neq 0$ & $3.92 \mathrm{E}-13$ & reject $H_{0}$ & $\beta_{L} \neq 0$ \\
\hline \multirow{4}{*}{ Madeira } & Flood & $\beta_{F}=0$ & $\beta_{F} \neq 0$ & $8.49 \mathrm{E}-13$ & reject $H_{0}$ & $\beta_{F} \neq 0$ \\
& Mean flow & $\beta_{M}=0$ & $\beta_{M} \neq 0$ & $9.17 \mathrm{E}-13$ & reject $H_{0}$ & $\beta_{M} \neq 0$ \\
& Low flow & $\beta_{L}=0$ & $\beta_{L} \neq 0$ & $2.32 \mathrm{E}-10$ & reject $H_{0}$ & $\beta_{L} \neq 0$ \\
\hline \multirow{4}{*}{ Tapajos } & Flood & $\beta_{F}=0$ & $\beta_{F} \neq 0$ & $8.41 \mathrm{E}-07$ & reject $H_{0}$ & $\beta_{F} \neq 0$ \\
& Mean flow & $\beta_{M}=0$ & $\beta_{M} \neq 0$ & $2.15 \mathrm{E}-07$ & reject $H_{0}$ & $\beta_{M} \neq 0$ \\
& Low flow & $\beta_{L}=0$ & $\beta_{L} \neq 0$ & $2.38 \mathrm{E}-05$ & reject $H_{0}$ & $\beta_{L} \neq 0$ \\
\hline \multirow{5}{*}{ Xingu } & Flood & $\beta_{F}=0$ & $\beta_{F} \neq 0$ & $1.00 \mathrm{E}-02$ & reject $H_{0}$ & $\beta_{F} \neq 0$ \\
& Mean flow & $\beta_{M}=0$ & $\beta_{M} \neq 0$ & $2.00 \mathrm{E}-03$ & reject $H_{0}$ & $\beta_{M} \neq 0$ \\
& Low flow & $\beta_{L}=0$ & $\beta_{L} \neq 0$ & $4.00 \mathrm{E}-02$ & reject $H_{0}$ & $\beta_{L} \neq 0$ \\
\hline
\end{tabular}

5 Tests for the difference between the values of the scaling exponents and the critical value 1 , using $L A$ as the scale parameter

Figure 3 indicates whether the values of the scaling exponents $\left(\beta_{i}, i=L, M, F\right)$ are significantly $(p<0.05)$ different, either higher or lower, from the critical value 1 . This is based on the $t$-tests results showed in Tables S3-S8. Why 1 is regarded as a critical value is explained in the main text. In the Xingu basin, the hypothesis that all exponents are equal to 1 cannot be rejected (this is indicated by the black dots in Fig. 3). This can be attributed to the small number of degrees of freedom given 
by the number of gauges within this basin. However, we keep some of the results for this river basin because the scaling relation given by Equation $(7)$ is still statistically significant $(p<0.05)$ for mean and extreme river flows (Table S2).

Table S3. $t$-tests for the difference between the critical value 1 and the scaling exponents for floods $\left(\beta_{F}\right)$, mean flows ( $\left.\beta_{M}\right)$, and low flows $\left(\beta_{L}\right)$ in the Amazon basin.

\begin{tabular}{lccccc}
\hline Q type & $H_{0}$ & $H_{a}$ & $p$-value & Test result & Conclusion \\
\hline \multirow{2}{*}{ Flood } & $\beta_{F}=1$ & $\beta_{F} \neq 1$ & $2.45 \mathrm{E}-08$ & reject $H_{0}$ & Floods are dampened, $\beta_{F}<1$ \\
& $\beta_{F} \geq 1$ & $\beta_{F}<1$ & $1.22 \mathrm{E}-08$ & reject $H_{0}$ & \\
\hline \multirow{2}{*}{ Mean flow } & $\beta_{M}=1$ & $\beta_{M} \neq 1$ & $\mathbf{9 . 7 9 E - 0 2}$ & do not reject $H_{0}$ & Mean flows are not amplified, $\beta_{M} \leq 1$ \\
& $\beta_{M} \geq 1$ & $\beta_{M}<1$ & $\mathbf{4 . 8 9 E - 0 2}$ & reject $H_{0}$ & \\
\hline \multirow{2}{*}{ Low flow } & $\beta_{L}=1$ & $\beta_{L} \neq 1$ & $2.00 \mathrm{E}-01$ & do not reject $H_{0}$ & Low flows are not dampened, $\beta_{L} \geq 1$ \\
& $\beta_{L} \geq 1$ & $\beta_{L}<1$ & $1.00 \mathrm{E}-01$ & do not reject $H_{0}$ & \\
\hline
\end{tabular}

Table S4. Same as Table S3 but for the Negro basin.

\begin{tabular}{lccccc}
\hline Q type & $H_{0}$ & $H_{a}$ & $p$-value & Test result & Conclusion \\
\hline \multirow{2}{*}{ Flood } & $\beta_{F}=1$ & $\beta_{F} \neq 1$ & $1.17 \mathrm{E}-03$ & reject $H_{0}$ & Floods are dampened, $\beta_{F}<1$ \\
& $\beta_{F} \geq 1$ & $\beta_{F}<1$ & $5.83 \mathrm{E}-04$ & reject $H_{0}$ & \\
\hline \multirow{2}{*}{ Mean flow } & $\beta_{M}=1$ & $\beta_{M} \neq 1$ & $8.26 \mathrm{E}-02$ & do not reject $H_{0}$ & Mean flows are not amplified, $\beta_{M} \leq 1$ \\
& $\beta_{M} \geq 1$ & $\beta_{M}<1$ & $4.13 \mathrm{E}-02$ & reject $H_{0}$ & \\
\hline \multirow{2}{*}{ Low flow } & $\beta_{L}=1$ & $\beta_{L} \neq 1$ & $9.45 \mathrm{E}-01$ & do not reject $H_{0}$ & Low flows are not dampened, $\beta_{L} \approx 1$ \\
& $\beta_{L} \geq 1$ & $\beta_{L}<1$ & $4.72 \mathrm{E}-01$ & do not reject $H_{0}$ & \\
\hline
\end{tabular}

Table S5. Same as Table S3 but for the Solimoes basin.

\begin{tabular}{lccccc}
\hline Q type & $H_{0}$ & $H_{a}$ & $p$-value & Test result & Conclusion \\
\hline \multirow{2}{*}{ Flood } & $\beta_{F}=1$ & $\beta_{F} \neq 1$ & $2.70 \mathrm{E}-01$ & do not reject $H_{0}$ & Floods are not amplified, $\beta_{F} \leq 1$ \\
& $\beta_{F} \leq 1$ & $\beta_{F}>1$ & $1.34 \mathrm{E}-01$ & do not reject $H_{0}$ & \\
\hline \multirow{2}{*}{ Mean flow } & $\beta_{M}=1$ & $\beta_{M} \neq 1$ & $1.34 \mathrm{E}-02$ & reject $H_{0}$ & Mean flows are amplified, $\beta_{M}>1$ \\
& $\beta_{M} \leq 1$ & $\beta_{M}>1$ & $6.74 \mathrm{E}-03$ & reject $H_{0}$ & \\
\hline \multirow{2}{*}{ Low flow } & $\beta_{L}=1$ & $\beta_{L} \neq 1$ & $5.53 \mathrm{E}-06$ & reject $H_{0}$ & Low flows are amplified, $\beta_{L}>1$ \\
& $\beta_{L} \leq 1$ & $\beta_{L}>1$ & $2.76 \mathrm{E}-06$ & reject $H_{0}$ & \\
\hline
\end{tabular}


Table S6. Same as Table S3 but for the Madeira basin.

\begin{tabular}{lccccc}
\hline Q type & $H_{0}$ & $H_{a}$ & $p$-value & Test result & Conclusion \\
\hline \multirow{2}{*}{ Flood } & $\beta_{F}=1$ & $\beta_{F} \neq 1$ & $1.33 \mathrm{E}-03$ & reject $H_{0}$ & Floods are dampened, $\beta_{F}<1$ \\
& $\beta_{F} \geq 1$ & $\beta_{F}<1$ & $6.65 \mathrm{E}-04$ & reject $H_{0}$ & \\
\hline \multirow{2}{*}{ Mean flow } & $\beta_{M}=1$ & $\beta_{M} \neq 1$ & $4.33 \mathrm{E}-02$ & reject $H_{0}$ & Mean flows are dampened, $\beta_{M}<1$ \\
& $\beta_{M} \geq 1$ & $\beta_{M}<1$ & $2.16 \mathrm{E}-02$ & reject $H_{0}$ & \\
\hline \multirow{2}{*}{ Low flow } & $\beta_{L}=1$ & $\beta_{L} \neq 1$ & $3.70 \mathrm{E}-01$ & do not reject $H_{0}$ & Low flows are not amplified, $\beta_{L} \leq 1$ \\
& $\beta_{L} \leq 1$ & $\beta_{L}>1$ & $1.85 \mathrm{E}-01$ & do not reject $H_{0}$ & \\
\hline
\end{tabular}

Table S7. Same as Table S3 but for the Tapajos basin.

\begin{tabular}{lccccc}
\hline Q type & $H_{0}$ & $H_{a}$ & $p$-value & Test result & Conclusion \\
\hline \multirow{2}{*}{ Flood } & $\beta_{F}=1$ & $\beta_{F} \neq 1$ & $1.26 \mathrm{E}-01$ & do not reject $H_{0}$ & Floods are not amplified, $\beta_{F} \leq 1$ \\
& $\beta_{F} \leq 1$ & $\beta_{F}>1$ & $6.29 \mathrm{E}-02$ & do not reject $H_{0}$ & \\
\hline \multirow{2}{*}{ Mean flow } & $\beta_{M}=1$ & $\beta_{M} \neq 1$ & $3.27 \mathrm{E}-02$ & reject $H_{0}$ & \\
& $\beta_{M} \geq 1$ & $\beta_{M}<1$ & $1.63 \mathrm{E}-02$ & reject $H_{0}$ & Mean flows are dampened, $\beta_{M}<1$ \\
\hline \multirow{2}{*}{ Low flow } & $\beta_{L}=1$ & $\beta_{L} \neq 1$ & $1.27 \mathrm{E}-02$ & reject $H_{0}$ & \\
& $\beta_{L} \geq 1$ & $\beta_{L}<1$ & $6.33 \mathrm{E}-03$ & reject $H_{0}$ & Low flows are dampened, $\beta_{L}<1$ \\
\hline
\end{tabular}

Table S8. Same as Table S3 but for the Xingu basin.

\begin{tabular}{lccccc}
\hline Q type & $H_{0}$ & $H_{a}$ & $p$-value & Test result & Conclusion \\
\hline \multirow{2}{*}{ Flood } & $\beta_{F}=1$ & $\beta_{F} \neq 1$ & $2.30 \mathrm{E}-01$ & do not reject $H_{0}$ & $\beta_{F} \approx 1$ \\
& $\beta_{F} \geq 1$ & $\beta_{F}<1$ & $1.10 \mathrm{E}-01$ & do not reject $H_{0}$ & \\
\hline \multirow{2}{*}{ Mean flow } & $\beta_{M}=1$ & $\beta_{M} \neq 1$ & $1.40 \mathrm{E}-01$ & do not reject $H_{0}$ & \multirow{2}{*}{$\approx$} \\
& $\beta_{M} \geq 1$ & $\beta_{M}<1$ & $7.00 \mathrm{E}-02$ & do not reject $H_{0}$ & \\
\hline \multirow{2}{*}{ Low flow } & $\beta_{L}=1$ & $\beta_{L} \neq 1$ & $3.80 \mathrm{E}-01$ & do not reject $H_{0}$ & \multirow{2}{*}{$\approx$} \\
& $\beta_{L} \leq 1$ & $\beta_{L}>1$ & $1.90 \mathrm{E}-01$ & do not reject $H_{0}$ & \\
\hline
\end{tabular}




\section{Scaling results using $A$ as the scale parameter}

This section presents the results of the scaling analysis when using $A$ instead of $L A$ as the scale parameter. Overall, the results are consistent between models. Here we use power law (hydrological scaling)

$$
E\left[Q_{i}\right]=\gamma_{i} A^{\delta_{i}}
$$

5 which is equivalent to Equation (1) with $S=A$ and $k=1$.

Table S9. $t$-tests for the significance of the regressions in the hydrological scaling for the first statistical moment of river flows. In all cases the null hypothesis, $H_{0}$ is rejected, so the alternative hypothesis, $H_{a}$, is accepted.

\begin{tabular}{llccccc}
\hline Basin & Q type & $H_{0}$ & $H_{a}$ & $p$-value & Test result & Conclusion \\
\hline \multirow{4}{*}{ Amazon } & Flood & $\delta_{F}=0$ & $\delta_{F} \neq 0$ & $<2.20 \mathrm{E}-16$ & reject $H_{0}$ & $\delta_{F} \neq 0$ \\
& Mean flow & $\delta_{M}=0$ & $\delta_{M} \neq 0$ & $<2.20 \mathrm{E}-16$ & reject $H_{0}$ & $\delta_{M} \neq 0$ \\
& Low flow & $\delta_{L}=0$ & $\delta_{L} \neq 0$ & $<2.20 \mathrm{E}-16$ & reject $H_{0}$ & $\delta_{L} \neq 0$ \\
\hline \multirow{3}{*}{ Negro } & Flood & $\delta_{F}=0$ & $\delta_{F} \neq 0$ & $9.14 \mathrm{E}-13$ & reject $H_{0}$ & $\delta_{F} \neq 0$ \\
& Mean flow & $\delta_{M}=0$ & $\delta_{M} \neq 0$ & $6.25 \mathrm{E}-10$ & reject $H_{0}$ & $\delta_{M} \neq 0$ \\
& Low flow & $\delta_{L}=0$ & $\delta_{L} \neq 0$ & $3.82 \mathrm{E}-06$ & reject $H_{0}$ & $\delta_{L} \neq 0$ \\
\hline \multirow{4}{*}{ Solimoes } & Flood & $\delta_{F}=0$ & $\delta_{F} \neq 0$ & $6.18 \mathrm{E}-11$ & reject $H_{0}$ & $\delta_{F} \neq 0$ \\
& Mean flow & $\delta_{M}=0$ & $\delta_{M} \neq 0$ & $7.68 \mathrm{E}-14$ & reject $H_{0}$ & $\delta_{M} \neq 0$ \\
& Low flow & $\delta_{L}=0$ & $\delta_{L} \neq 0$ & $5.57 \mathrm{E}-13$ & reject $H_{0}$ & $\delta_{L} \neq 0$ \\
\hline \multirow{4}{*}{ Madeira } & Flood & $\delta_{F}=0$ & $\delta_{F} \neq 0$ & $8.96 \mathrm{E}-11$ & reject $H_{0}$ & $\delta_{F} \neq 0$ \\
& Mean flow & $\delta_{M}=0$ & $\delta_{M} \neq 0$ & $1.93 \mathrm{E}-12$ & reject $H_{0}$ & $\delta_{M} \neq 0$ \\
& Low flow & $\delta_{L}=0$ & $\delta_{L} \neq 0$ & $2.58 \mathrm{E}-11$ & reject $H_{0}$ & $\delta_{L} \neq 0$ \\
\hline \multirow{4}{*}{ Tapajos } & Flood & $\delta_{F}=0$ & $\delta_{F} \neq 0$ & $4.78 \mathrm{E}-09$ & reject $H_{0}$ & $\delta_{F} \neq 0$ \\
& Mean flow & $\delta_{M}=0$ & $\delta_{M} \neq 0$ & $6.62 \mathrm{E}-09$ & reject $H_{0}$ & $\delta_{M} \neq 0$ \\
& Low flow & $\delta_{L}=0$ & $\delta_{L} \neq 0$ & $1.97 \mathrm{E}-05$ & reject $H_{0}$ & $\delta_{L} \neq 0$ \\
\hline \multirow{5}{*}{ Xingu } & Flood & $\delta_{F}=0$ & $\delta_{F} \neq 0$ & $1.10 \mathrm{E}-02$ & reject $H_{0}$ & $\delta_{F} \neq 0$ \\
& Mean flow & $\delta_{M}=0$ & $\delta_{M} \neq 0$ & $1.64 \mathrm{E}-03$ & reject $H_{0}$ & $\delta_{M} \neq 0$ \\
& Low flow & $\delta_{L}=0$ & $\delta_{L} \neq 0$ & $3.10 \mathrm{E}-02$ & reject $H_{0}$ & $\delta_{L} \neq 0$ \\
\hline
\end{tabular}



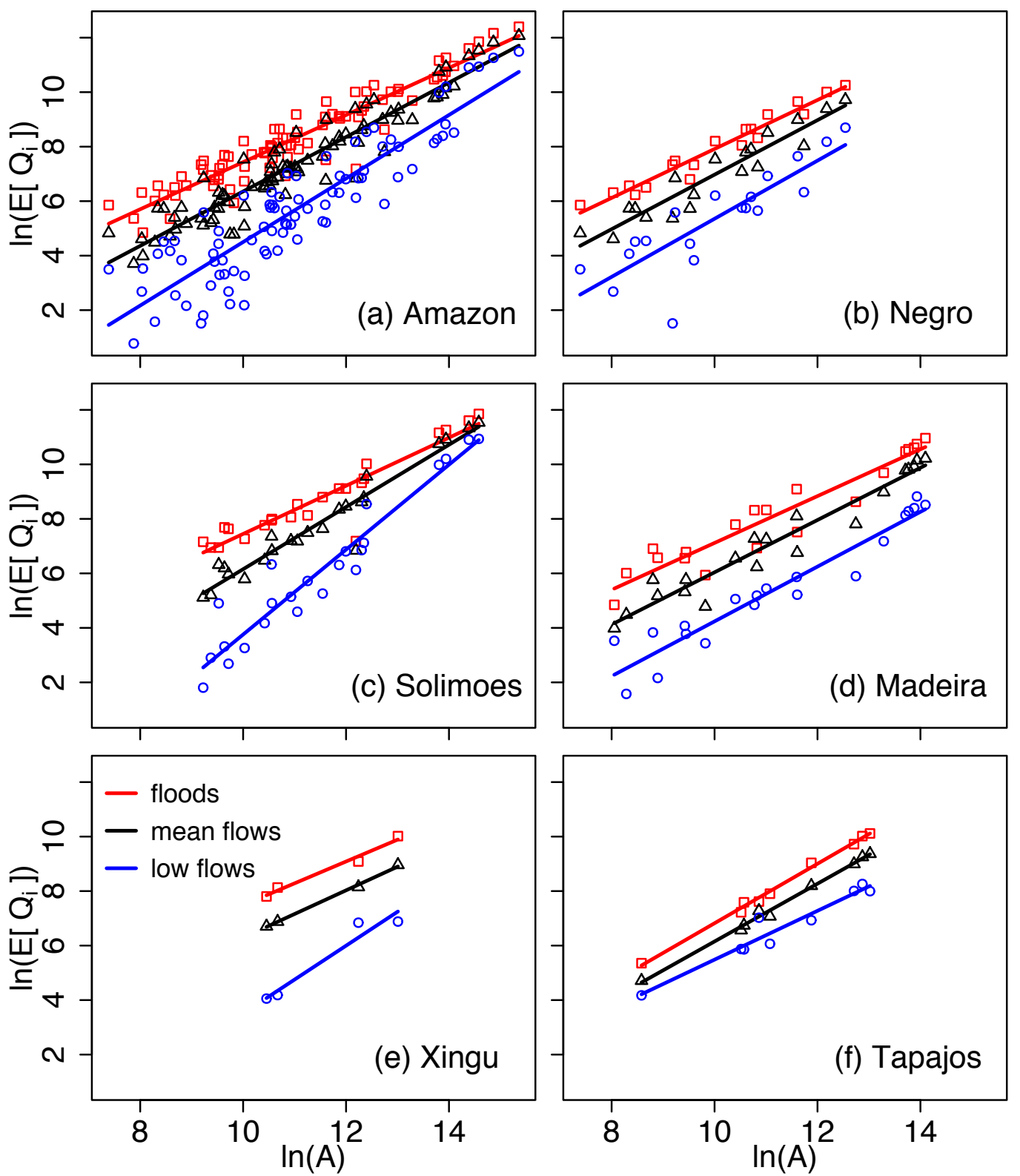

Figure S7. Same as Fig. 2 but using $A$ instead of $L A$. Power laws of the form $E[Q]=\gamma_{i} A^{\delta_{i}}$ (equivalent to Equation (1) with $S=A$ and $k=1)$ for low flows $(i=L)$, mean flows $(i=M)$, and floods $(i=F)$. Points are observed river flows and lines are the scaling relations (in all cases $r>0.88$ and $p<0.05)$. (a) Amazon: $E\left[Q_{L}\right]=\exp (-7.16) L A^{1.17} ; E\left[Q_{M}\right]=\exp (-3.63) L A^{1.00}$; $E\left[Q_{F}\right]=\exp (-1.22) L A^{0.86}$. (b) Negro: $E\left[Q_{L}\right]=\exp (-5.31) L A^{1.07} ; E\left[Q_{M}\right]=\exp (-3.03) L A^{1.00} ; E\left[Q_{F}\right]=\exp (-1.07) L A^{0.90}$. (c) Solimoes: $E\left[Q_{L}\right]=\exp (-11.83) L A^{1.55} ; E\left[Q_{M}\right]=\exp (-5.26) L A^{1.14} ; E\left[Q_{F}\right]=\exp (-1.38) L A^{0.88}$. (d) Madeira: $E\left[Q_{L}\right]=$ $\exp (-5.81) L A^{1.00} ; E\left[Q_{M}\right]=\exp (-3.55) L A^{0.96} ; E\left[Q_{F}\right]=\exp (-1.48) L A^{0.86}$. (e) Xingu: $E\left[Q_{L}\right]=\exp (-8.93) L A^{1.24} ; E\left[Q_{M}\right]=$ $\exp (-2.39) L A^{0.86} ; E\left[Q_{F}\right]=\exp (-0.53) L A^{0.80}$. (f) Tapajos: $E\left[Q_{L}\right]=\exp (-3.47) L A^{0.89} ; E\left[Q_{M}\right]=\exp (-4.45) L A^{1.06} ; E\left[Q_{F}\right]=$ $\exp (-4.09) L A^{1.09}$. For convenience, $\gamma_{i}$ is expressed as $\exp \left(\ln \left(\gamma_{i}\right)\right)$. 

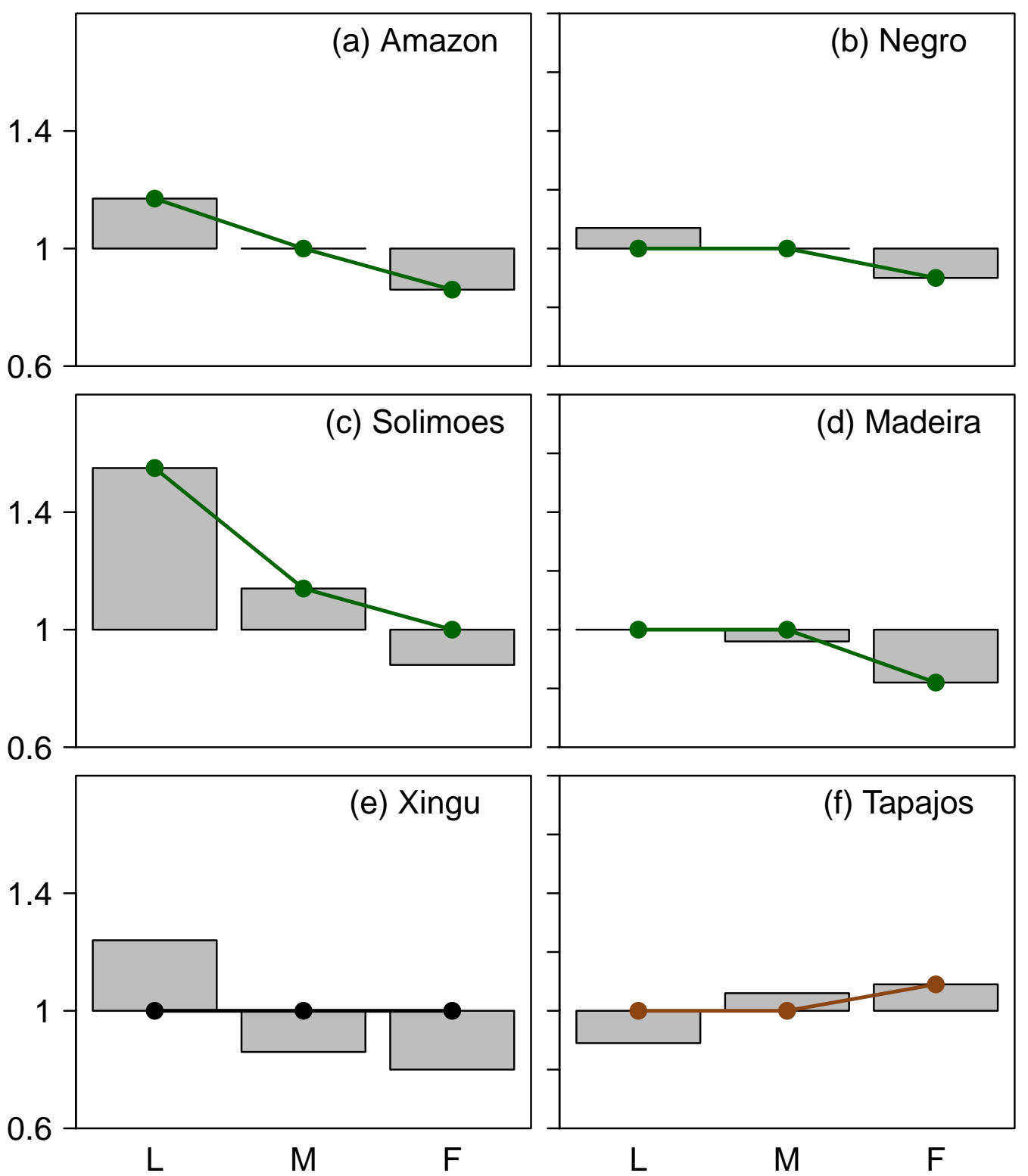

Figure S8. Same as Fig. 3 but using $A$ instead of $L A$. Observed patterns of the values of the scaling exponents $\left(\delta_{i}\right)$ for low flows (L), mean flows (M), and floods (F), in the Amazon basin and its six major sub-basins. Dots over the bars indicate whether the difference between the scaling exponent and 1 is significant ( $p<0.05$, the dot is not over 1 ) or not (the dot is over 1 ). Details about the $t$-tests are in Supplementary Tables S3 to S8. In regulated states (green, a-d), the exponents decrease from low flows to floods; whereas in unregulated states (brown, f), the exponents increase from low flows to floods. In the Xingu river basin (e), the hypothesis that all exponents are equal to 1 can not be rejected $(p>0.05)$ because of the small number of degrees of freedom (gauges). 

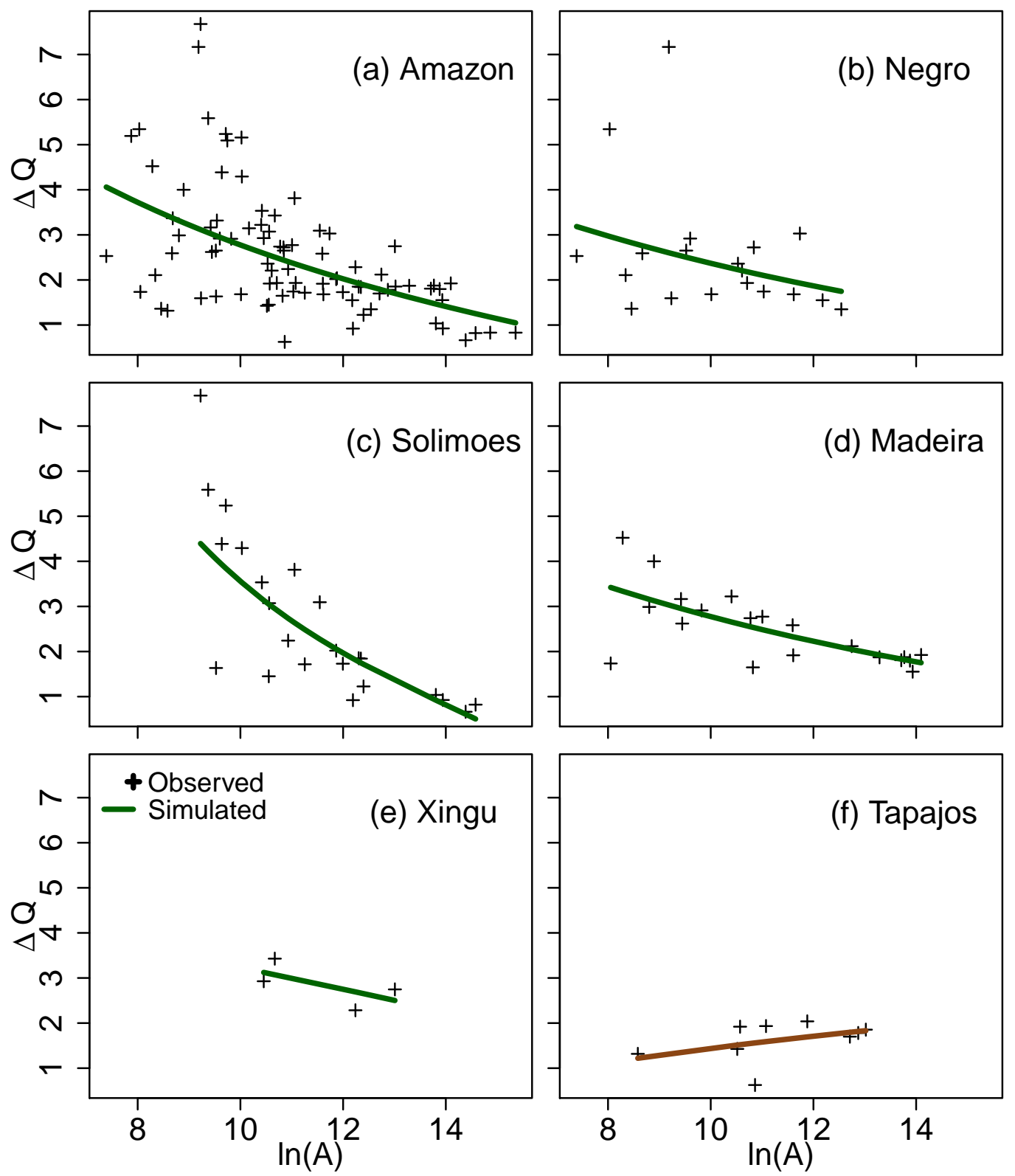

Figure S9. Same as Fig. 4 but using $A$ instead of $L A$. Extremes amplitude, $\Delta_{Q}=\left(E\left[Q_{F}\right]-E\left[Q_{L}\right]\right) / E\left[Q_{M}\right]$, as observed (crosses) and simulated (lines) by $\left(\gamma_{F} A^{\delta_{F}}-\gamma_{L} A^{\delta_{L}}\right) / \gamma_{M} A^{\delta_{M}}$ (from Eq. 2 with $S=A$ ), using the scaling parameters of each basin. $\Delta_{Q}$ either decreases or increases with spatial scale $(A)$ depending on whether the river basin is regulated ( $\delta_{L}>\delta_{M}>\delta_{F}$, e.g. Solimoes) or unregulated $\left(\delta_{L}<\delta_{M}<\delta_{F}\right.$, e.g. Tapajos). 
Table S10. Same as Table S3 but using $A$ instead of $L A$. $t$-tests for the difference between the critical value 1 and the scaling exponents for floods $\left(\delta_{F}\right)$, mean flows $\left(\delta_{M}\right)$, and low flows $\left(\delta_{L}\right)$ in the Amazon basin.

\begin{tabular}{lccccc}
\hline Q type & $H_{0}$ & $H_{a}$ & $p$-value & Test result & Conclusion \\
\hline \multirow{2}{*}{ Flood } & $\delta_{F}=1$ & $\delta_{F} \neq 1$ & $7.70 \mathrm{E}-05$ & reject $H_{0}$ & Floods are dampened, $\delta_{F}<1$ \\
& $\delta_{F} \geq 1$ & $\delta_{F}<1$ & $3.85 \mathrm{E}-05$ & reject $H_{0}$ & \\
\hline \multirow{2}{*}{ Mean flow } & $\delta_{M}=1$ & $\delta_{M} \neq 1$ & $9.75 \mathrm{E}-01$ & do not reject $H_{0}$ & $\delta_{M} \approx 1$ \\
& $\delta_{M} \geq 1$ & $\delta_{M}<1$ & $4.87 \mathrm{E}-01$ & do not reject $H_{0}$ & \\
\hline \multirow{2}{*}{ Low flow } & $\delta_{L}=1$ & $\delta_{L} \neq 1$ & $9.27 \mathrm{E}-03$ & reject $H_{0}$ & Low flows are amplified $\delta_{L}>1$ \\
\hline
\end{tabular}

Table S11. Same as Table S10 but for the Negro basin.

\begin{tabular}{lccccc}
\hline Q type & $H_{0}$ & $H_{a}$ & $p$-value & Test result & Conclusion \\
\hline \multirow{2}{*}{ Flood } & $\delta_{F}=1$ & $\delta_{F} \neq 1$ & $4.97 \mathrm{E}-02$ & reject $H_{0}$ & Floods are dampened, $\delta_{F}<1$ \\
& $\delta_{F} \geq 1$ & $\delta_{F}<1$ & $2.49 \mathrm{E}-02$ & reject $H_{0}$ & \\
\hline \multirow{2}{*}{ Mean flow } & $\delta_{M}=1$ & $\delta_{M} \neq 1$ & $9.97 \mathrm{E}-01$ & do not reject $H_{0}$ & \\
& $\delta_{M} \geq 1$ & $\delta_{M}<1$ & $4.99 \mathrm{E}-01$ & do not reject $H_{0}$ & \\
\hline \multirow{2}{*}{ Low flow } & $\delta_{L}=1$ & $\delta_{L} \neq 1$ & $6.83 \mathrm{E}-01$ & do not reject $H_{0}$ & Low flows are not dampened, $\delta_{L} \geq 1$ \\
\hline
\end{tabular}

Table S12. Same as Table S10 but for the Solimoes basin.

\begin{tabular}{lccccc}
\hline Q type & $H_{0}$ & $H_{a}$ & $p$-value & Test result & Conclusion \\
\hline \multirow{2}{*}{ Flood } & $\delta_{F}=1$ & $\delta_{F} \neq 1$ & $1.24 \mathrm{E}-01$ & do not reject $H_{0}$ & Floods are not amplified, $\delta_{F} \leq 1$ \\
& $\delta_{F} \leq 1$ & $\delta_{F}>1$ & $6.22 \mathrm{E}-02$ & do not reject $H_{0}$ & \\
\hline \multirow{2}{*}{ Mean flow } & $\delta_{M}=1$ & $\delta_{M} \neq 1$ & $4.51 \mathrm{E}-02$ & reject $H_{0}$ & Mean flows are amplified, $\delta_{M}>1$ \\
& $\delta_{M} \leq 1$ & $\delta_{M}>1$ & $2.25 \mathrm{E}-02$ & reject $H_{0}$ & \\
\hline \multirow{2}{*}{ Low flow } & $\delta_{L}=1$ & $\delta_{L} \neq 1$ & $1.62 \mathrm{E}-05$ & reject $H_{0}$ & Low flows are amplified, $\delta_{L}>1$ \\
& $\delta_{L} \leq 1$ & $\delta_{L}>1$ & $8.09 \mathrm{E}-06$ & reject $H_{0}$ & \\
\hline
\end{tabular}


Table S13. Same as Table S10 but for the Madeira basin.

\begin{tabular}{lccccc}
\hline Q type & $H_{0}$ & $H_{a}$ & $p$-value & Test result & Conclusion \\
\hline \multirow{2}{*}{ Flood } & $\delta_{F}=1$ & $\delta_{F} \neq 1$ & $4.22 \mathrm{E}-02$ & reject $H_{0}$ & Floods are dampened, $\delta_{F}<1$ \\
& $\delta_{F} \geq 1$ & $\delta_{F}<1$ & $2.11 \mathrm{E}-02$ & reject $H_{0}$ & \\
\hline \multirow{2}{*}{ Mean flow } & $\delta_{M}=1$ & $\delta_{M} \neq 1$ & $4.85 \mathrm{E}-01$ & do not reject $H_{0}$ & Mean flows are not amplified, $\delta_{M} \leq 1$ \\
& $\delta_{M} \leq 1$ & $\delta_{M}>1$ & $2.43 \mathrm{E}-01$ & do not reject $H_{0}$ & \\
\hline \multirow{2}{*}{ Low flow } & $\delta_{L}=1$ & $\delta_{L} \neq 1$ & $9.45 \mathrm{E}-01$ & do not reject $H_{0}$ & \\
& $\delta_{L} \geq 1$ & $\delta_{L}<1$ & $4.73 \mathrm{E}-01$ & do not reject $H_{0}$ & \\
\hline
\end{tabular}

Table S14. Same as Table S10 but for the Tapajos basin.

\begin{tabular}{lccccc}
\hline Q type & $H_{0}$ & $H_{a}$ & $p$-value & Test result & Conclusion \\
\hline \multirow{2}{*}{ Flood } & $\delta_{F}=1$ & $\delta_{F} \neq 1$ & $2.46 \mathrm{E}-02$ & reject $H_{0}$ & Floods are amplified, $\delta_{F}>1$ \\
& $\delta_{F} \leq 1$ & $\delta_{F}>1$ & $1.23 \mathrm{E}-02$ & reject $H_{0}$ & \\
\hline \multirow{2}{*}{ Mean flow } & $\delta_{M}=1$ & $\delta_{M} \neq 1$ & $1.07 \mathrm{E}-01$ & do not reject $H_{0}$ & Mean flows are not amplified, $\delta_{M} \leq 1$ \\
& $\delta_{M} \leq 1$ & $\delta_{M}>1$ & $5.37 \mathrm{E}-02$ & do not reject $H_{0}$ & \\
\hline \multirow{2}{*}{ Low flow } & $\delta_{L}=1$ & $\delta_{L} \neq 1$ & $2.77 \mathrm{E}-01$ & do not reject $H_{0}$ & Low flows are not amplified, $\delta_{L} \leq 1$ \\
& $\delta_{L} \leq 1$ & $\delta_{L}>1$ & $1.38 \mathrm{E}-01$ & do not reject $H_{0}$ & \\
\hline
\end{tabular}

Table S15. Same as Table S10 but for the Xingu basin.

\begin{tabular}{lccccc}
\hline Q type & $H_{0}$ & $H_{a}$ & $p$-value & Test result & Conclusion \\
\hline \multirow{2}{*}{ Flood } & $\delta_{F}=1$ & $\delta_{F} \neq 1$ & $1.44 \mathrm{E}-01$ & do not reject $H_{0}$ & $\delta_{F} \approx 1$ \\
& $\delta_{F} \geq 1$ & $\delta_{F}<1$ & $7.19 \mathrm{E}-02$ & do not reject $H_{0}$ & \\
\hline \multirow{2}{*}{ Mean flow } & $\delta_{M}=1$ & $\delta_{M} \neq 1$ & $6.44 \mathrm{E}-02$ & do not reject $H_{0}$ & \multirow{2}{*}{$\delta_{M} \approx 1$} \\
& $\delta_{M} \geq 1$ & $\delta_{M}<1$ & $3.22 \mathrm{E}-02$ & reject $H_{0}$ & \\
\hline \multirow{2}{*}{ Low flow } & $\delta_{L}=1$ & $\delta_{L} \neq 1$ & $3.91 \mathrm{E}-01$ & do not reject $H_{0}$ & \multirow{2}{*}{$\delta_{L} \approx 1$} \\
& $\delta_{L} \leq 1$ & $\delta_{L}>1$ & $1.95 \mathrm{E}-01$ & do not reject $H_{0}$ & \\
\hline
\end{tabular}

